\title{
FREE VIBRATION ANALYSIS OF THICK DISKS WITH VARIABLE THICKNESS CONTAINING ORTHOTROPIC-NONHOMOGENEOUS MATERIAL USING FINITE ELEMENT METHOD
}

\author{
Hassan Zafarmand, Mehran Kadkhodayan \\ Ferdowsi University of Mashhad, Department of Mechanical Engineering, Mashhad, Iran \\ e-mail: kadkhoda@um.ac.ir
}

\begin{abstract}
Free vibration analysis of thick functionally graded nanocomposite annular and solid disks with variable thickness reinforced by single-walled carbon nanotubes (SWCNTs) is presented. Four types of distribution of uniaxial aligned SWCNTs are considered: uniform and three kinds of functionally graded (FG) distribution through radial direction of the disk. The effective material properties of the nanocomposite disk are estimated by a micro mechanical model. The axisymmetric conditions are assumed and employing the graded finite element method (GFEM), the equations are solved. The solution is considered for four different thickness profiles, namely constant, linear, concave and convex. The achieved results show that the type of distribution and volume fraction of CNTs and thickness profile have a great effect on normalized natural frequencies.
\end{abstract}

Keywords: free vibration, carbon nanotube, functionally graded material, thick disk, variable thickness, graded finite element method

\section{Introduction}

In the recent years, nano-structured materials such as nanocomposites, have generated considerable interest in the material research community and became an attractive new subject in material science due to their potentially impressive mechanical properties. Carbon nanotubes (CNTs) have illustrated remarkable mechanical, thermal and electrical properties. For example, they could potentially have a Young's modulus as high as $1 \mathrm{TPa}$ and a tensile strength approaching $100 \mathrm{GPa}$ (Odegard et al., 2002). These enormous advantages make them highly desirable candidates for the reinforcement of polymer composites, provided that good interfacial bonding between CNTs and polymer and proper dispersion of the individual CNTs in the polymeric matrix can be assured (Fiedler et al., 2006).

The majority of researches performed on carbon nanotube reinforced composites (CNTRCs) are focused on their material properties (Esawi and Farag, 2007; Thostenson et al., 2001; Dai, 2006; Kang et al., 2006; Lau et al., 2006). Han and Elliott (2007), by the use of molecular dynamic simulation (MD), obtained the elastic modulus of composite structures reinforced by CNTs and studied the effect of volume fraction of SWCNTs on mechanical properties of nanocomposites. $\mathrm{Hu}$ et al. (2005), by analyzing the elastic deformation of a representative volume element (RVE) under various loading conditions, evaluated the macroscopic elastic properties of CNTRCs. Zhu et al. (2007) studied the effect of CNTs on the mechanical properties of polymeric composites. Their results showed that adding CNTs could greatly improve Young's modulus. Due to dependency of the interaction at the polymer and nanotube interface on the local molecular structure and bonding, Odegard et al. (2003) a constitutive model for CNTRCs by utilizing an equivalent-continuum modeling method proposed.

Functionally graded materials (FGMs) are special composite materials, microscopically inhomogeneous, in which mechanical properties vary smoothly and continuously from one surface 
to the other. This idea was used for the first time by Japanese researchers (Koizumi, 1993) and led to the concept of FGMs. A wide range of researches have been carried out on FGMs in various fields of mechanics. Motivated by the concept of FGMs, Shen (2009) presented a kind of CNTRCs in which the volume fraction of CNTs is graded with certain rules through desired directions and demonstrated that using FG-CNTRCs improve the mechanical properties of the structures. Zhu et al. (2012) studied bending and free vibration analyses of composite plates reinforced by SWCNTs using the finite element method based on the first order shear deformation plate theory. The effective material properties of the FG-CNTRC are graded in the thickness direction and are estimated according to the rule of mixture. Zafarmand and Kadkhodayan (2015) investigated nonlinear behaviour of FG-CNTRC rotating thick disks with variable thickness where the nonlinear axisymmetric theory of elasticity is employed. The three dimensional free vibration analysis of FC-CNTRC panels was investigated by Yas et al. (2013). The boundary conditions were assumed to be simply supported and the equations were solved by a generalized differential quadrature (GDQ) method. Ke et al. (2010) performed nonlinear free vibration analysis of FG-CNTRC beams based on Timoshenko beam theory and Von-Karman geometric nonlinearity. The Ritz method was applied to derive the governing eigenvalue equation which was then solved by a direct iterative method to obtain the nonlinear vibration frequencies of FG-CNTRC beams with different end supports. Sobhani Aragh and Yas (2010) investigated the static and free vibration characteristics of continuously graded fiber-reinforced (CGFR) cylindrical shells based on three dimensional elasticity. The boundary conditions were assumed to be simply supported and the equations were solved by a GDQ method. Moreover, several researches were carried out about free vibration analysis of FG disks with variable thickness (Alipour et al., 2010; Gupta et al., 2007; Efraim and Eisenberger, 2007; Tajeddini and Ohadi, 2011).

The purpose of this paper is to investigate free vibration analysis of thick FG-CNTRC annular and solid disks with variable thickness. Material properties are assumed to vary continuously through radial direction. The effective material properties of FG-CNTRC disks are estimated using a micro-mechanical model and the normalized natural frequencies of FG-CNTRC annular and solid disks for various types of distributions and volume fractions of CNTs, boundary conditions and thickness to radius ratios as well as different kinds of thickness profiles are computed and compared. The difficulty in obtaining analytical solutions for the response of graded material systems is due to the dispersive nature of heterogeneous material systems. Therefore, analytical or semi-analytical solutions are available only through a number of problems with simple boundary conditions. Thus, in order to find the solution for a thick FG-CNTRC disk of variable thickness with various boundary conditions, powerful numerical methods such as the graded finite element method (GFEM) are needed. The graded finite element incorporates the gradient of material properties at the element scale in the framework of a generalized isoparametric formulation. Some studies can be found in the literature on the modeling of non-homogenous structures by using GFEM (Kim and Paulino, 2002; Zafarmand and Hassani, 2014; Ashrafi et al., 2013).

\section{Problem formulation}

In this Section, different types of CNTs distributions through radial direction of the disk is introduced. The axisymmetric governing equations of motion are obtained and the graded finite element method is used for modeling the non-homogeneity of the material.

\subsection{Material properties in FG-CNTRC disks}

A thick FG-CNTRC disk of inner radius $a$, outer radius $b$ and variable thickness $h(r)$ is considered. The geometry and coordinate system of the disk is shown in Fig. 1. 


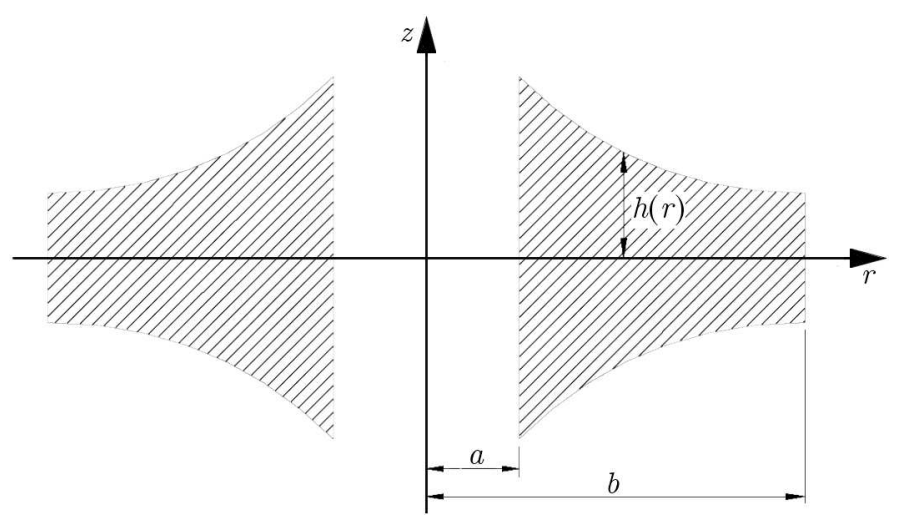

Fig. 1. Axisymmetric thick FG-CNTRC disk

This FG-CNTRC disk consists of SWCNTs (along the radial direction) and an isotropic matrix. UD-CNTRC represents the uniform distribution and FG_V, FG_O and FG_X-CNTRC, the functionally graded distribution of CNTs in the radial direction of the nanocomposite disk. Several studies have been published each with different focuses on mechanical properties of CNTRCs. However, due to the simplicity and convenience, in the present study, the rule of mixture is employed, and thus the effective material properties of CNTRC disk can be obtained as (Shen, 2009)

$$
\begin{array}{ll}
E_{1}=\eta_{1} V_{C N T} E_{1}^{C N T}+V_{m} E^{m} & \frac{\eta_{2}}{E_{i}}=\frac{V_{C N T}}{E_{i}^{C N T}}+\frac{V_{m}}{E^{m}} \quad(i=2,3) \\
\frac{\eta_{3}}{G_{i j}}=\frac{V_{C N T}}{G_{i j}^{C N T}}+\frac{V_{m}}{G^{m}} & \nu_{i j}=V_{C N T} \nu_{i j}^{C N T}+V_{m} \nu^{m} \\
\rho=V_{C N T} \rho^{C N T}+V_{m} \rho^{m} &
\end{array} \quad(i \neq j)
$$

where $E_{i}^{C N T}, G_{i j}^{C N T}, \nu_{i j}^{C N T}$ and $\rho^{C N T}$ are elasticity modulus, shear modulus, Poisson's ratio and density, respectively, of the CNTs, and $E^{m}, G^{m}, \nu^{m}$ and $\rho^{m}$ are the corresponding properties of the matrix. $\eta_{j}(j=1,2,3)$ is the CNTs' efficiency parameter which can be computed by matching the elastic modulus of CNTRCs observed from the MD simulation results with those obtained from the rule of mixture. $V_{C N T}$ and $V_{m}$ are volume fractions of the CNTs and matrix, respectively, which are related by $V_{C N T}+V_{m}=1$. The type of distribution and volume fraction of the CNTs has serious effects on the disk behavior. As has been mentioned previously, four types of distribution are utilized in this study; that is either uniformly distributed (UD) or functionally graded (FG) in the radial direction of the disk. These types with the distribution for a section in the $r-z$ plane of the disk with a constant thickness profile are depicted in Fig. 2 (the dash lines are CNTs in a huge scale). Distributions of CNTs through the radial direction of these four types of CNTRC disks are assumed to be as (Shen, 2009)

$$
\begin{aligned}
V_{C N T} & =V_{C N T}^{*} & & \text { type UD } \\
V_{C N T} & =2 \frac{r-a}{b-a} V_{C N T}^{*} & & \text { type FG_V } \\
V_{C N T} & =2 \frac{b-r}{b-a} V_{C N T}^{*} & & \text { type FG_O } \\
V_{C N T} & =4\left|\frac{r-a}{b-a}\right| V_{C N T}^{*} & & \text { type FG_X }
\end{aligned}
$$

in which

$$
r_{m}=\frac{a+b}{2} \quad V_{C N T}^{*}=\frac{w_{C N T}}{w_{C N T}+\frac{\rho_{C N T}}{\rho_{m}}\left(1-w_{C N T}\right)}
$$


where $w_{C N T}$ is the mass fraction of CNTs which does not depend on the variable $r$ and is unique for all types of distribution. $\rho_{C N T}$ and $\rho_{m}$ are the densities of CNTs and matrix, respectively.
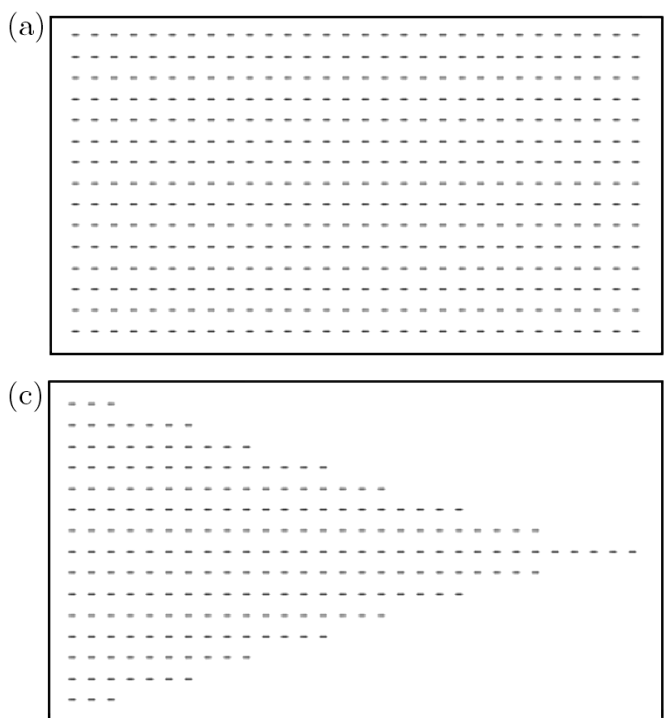

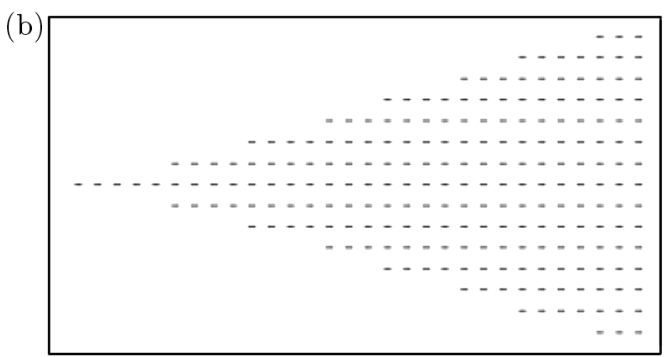

(d)

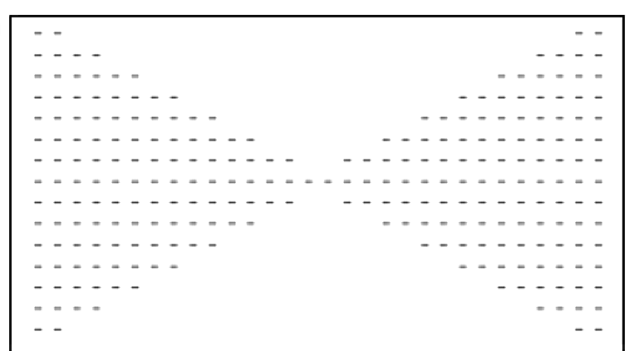

Fig. 2. Different types of distribution: (a) UD, (b) FG_V, (c) FG_O, (d) FG_X

\subsection{Governing equations}

The governing equations may be obtained based on Hamilton's principle

$$
\int_{t_{1}}^{t_{2}} \delta I d t=\int_{t_{1}}^{t_{2}} \delta(\Pi-T) d t=0
$$

where $\Pi$ and $T$ are potential and kinetic energy, respectively. These functions and their variations are

$$
\begin{array}{ll}
\Pi=\frac{1}{2} \iiint_{\Omega} \varepsilon^{\mathrm{T}} \cdot \boldsymbol{\sigma} d \Omega & \delta \Pi=\frac{1}{2} \iiint_{\Omega} \delta \varepsilon^{\mathrm{T}} \cdot \boldsymbol{\sigma} d \Omega \\
T=\frac{1}{2} \iiint_{\Omega} \rho \dot{\mathbf{U}}^{\mathrm{T}} \cdot \dot{\mathbf{U}} d \Omega & \delta T=\frac{1}{2} \iiint_{\Omega} \rho \dot{\mathbf{U}}^{\mathrm{T}} \cdot \delta \dot{\mathbf{U}} d \Omega
\end{array}
$$

where $\Omega$ is the volume of the domain under consideration and $\rho$ is the mass density that depends on the $r$ coordinate.

Substituting Eqs. (2.5) into Hamilton's principle (Eq. (2.4)), applying the side conditions $\left.\delta \mathbf{U}\right|_{t_{1}, t_{2}}=\mathbf{0}$ and using part integration, one obtains

$$
\iiint_{\Omega} \delta \varepsilon^{\mathrm{T}} \cdot \boldsymbol{\sigma} d \Omega+\iiint_{\Omega} \rho \ddot{\mathbf{U}}^{\mathrm{T}} \cdot \delta \dot{\mathbf{U}} d \Omega=0
$$

The stress-strain relations from Hook's law in matrix form are as

$$
\sigma=\mathbf{D} \varepsilon
$$

where the stress and strain components and the coefficients of elasticity $\mathbf{D}$ are as in the following relations 


$$
\begin{aligned}
& \boldsymbol{\sigma}=\left\{\begin{array}{llll}
\sigma_{r} & \sigma_{\theta} & \sigma_{z} & \sigma_{r z}
\end{array}\right\}^{\mathrm{T}} \quad \boldsymbol{\varepsilon}=\left\{\begin{array}{llll}
\varepsilon_{r} & \varepsilon_{\theta} & \varepsilon_{z} & \gamma_{r z}
\end{array}\right\}^{\mathrm{T}} \\
& \mathbf{D}=\left[\begin{array}{cccc}
D_{11} & D_{12} & D_{13} & 0 \\
D_{12} & D_{22} & D_{23} & 0 \\
D_{13} & D_{23} & D_{33} & 0 \\
0 & 0 & 0 & D_{55}
\end{array}\right]
\end{aligned}
$$

in which

$$
\begin{aligned}
& D_{11}=\frac{1-\nu_{23} \nu_{32}}{E_{2} E_{3} \Delta} \quad D_{22}=\frac{1-\nu_{13} \nu_{31}}{E_{1} E_{3} \Delta} \quad D_{33}=\frac{1-\nu_{12} \nu_{21}}{E_{1} E_{2} \Delta} \\
& D_{12}=\frac{\nu_{21}+\nu_{31} \nu_{23}}{E_{2} E_{3} \Delta} \quad D_{13}=\frac{\nu_{31}+\nu_{21} \nu_{32}}{E_{2} E_{3} \Delta} \quad D_{23}=\frac{\nu_{32}+\nu_{12} \nu_{31}}{E_{1} E_{3} \Delta} \\
& D_{55}=G_{13} \quad \Delta=\frac{1-\nu_{12} \nu_{21}-\nu_{23} \nu_{32}-\nu_{13} \nu_{31}-2 \nu_{21} \nu_{32} \nu_{13}}{E_{1} E_{2} E_{3}}
\end{aligned}
$$

where $E_{i}, G_{i j}$ and $\nu_{i j}$ are found from (2.1). It is obvious that the matrix $\mathbf{D}$ is dependent on the spatial variable $r$.

The strain-displacement equations based on the theory of linear theory of elasticity in cylindrical coordinates with the axisymmetric assumption are

$$
\varepsilon_{r}=\frac{\partial u}{\partial r} \quad \varepsilon_{\theta}=\frac{u}{r} \quad \varepsilon_{z}=\frac{\partial w}{\partial z} \quad \gamma_{r z}=\frac{\partial u}{\partial z}+\frac{\partial w}{\partial r}
$$

where $u$ and $w$ are the radial and axial components of the displacement, respectively. Equation (2.10) can be formulated in the matrix form as

$$
\varepsilon=\mathbf{L U}
$$

in which $\mathbf{U}$ is the displacements vector and $\mathbf{L}$ is a matrix containing partial differentiating equations as

$$
\mathbf{U}=\left\{\begin{array}{ll}
u & v
\end{array}\right\}^{\mathrm{T}} \quad \mathbf{L}=\left[\begin{array}{cccc}
\partial_{r} & 1 / r & 0 & \partial_{z} \\
0 & 0 & \partial_{z} & \partial_{r}
\end{array}\right]^{\mathrm{T}}
$$

Moreover, the boundary conditions used in this study are defined as follows: Solid disk:

- Clamped

- Simply supported

- Free

Annular disk:

- Clamped

- Simply supported

- Free

$$
\begin{array}{lll}
r=b & \rightarrow & u=w=0 \\
r=b & \rightarrow & \sigma_{r}=w=0 \\
r=b & \rightarrow & \sigma_{r}=\sigma_{r z}=0
\end{array}
$$

\subsection{Graded finite element modeling}

In order to solve the governing equations, the isoparametric finite element method with graded element properties is employed. For this purpose, the variational formulation is considered. In conventional finite element formulations, a predetermined set of material properties are used for each element such that the property field is constant within an individual element. For modeling a continuously nonhomogeneous material, the material property function must be 
discretized according to the size of elements mesh. This approximation can provide significant discontinuities. Based on these facts, the graded finite element is strongly preferable for the modeling of the present problem. Using the graded elements for the modeling of gradation of the material leads to more accurate results than dividing the solution domain into homogenous elements.

The finite element approximation of the domain is in the $r$-z plane, which is the plane of revolution. The section of the cylinder in the $r-z$ plane is considered and divided into a number of simplex linear quadrilateral elements. For convenience, we use the local coordinate with its variables $(\xi, \eta)$ between -1 to 1 , as shown in Fig. 3.

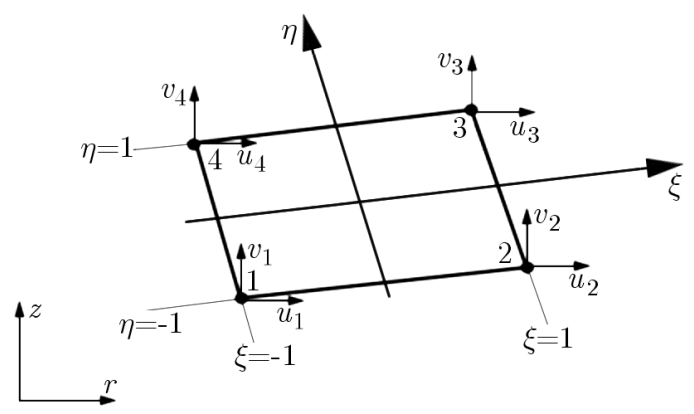

Fig. 3. Local coordinate

For element (e), the displacements are approximated as (Zienkiewicz, 2005)

$$
\mathbf{U}^{(e)}=\mathbf{\Phi} \Lambda^{(e)}
$$

where $\boldsymbol{\Phi}$ is the matrix of linear shape functions in the local coordinate and $\boldsymbol{\Lambda}^{(e)}$ is the nodal displacement vector of the element, which is

$$
\begin{aligned}
& \boldsymbol{\Phi}=\left[\begin{array}{cccccccc}
\Phi_{1} & 0 & \Phi_{2} & 0 & \Phi_{3} & 0 & \Phi_{4} & 0 \\
0 & \Phi_{1} & 0 & \Phi_{2} & 0 & \Phi_{3} & 0 & \Phi_{4}
\end{array}\right] \\
& \boldsymbol{\Lambda}^{(e)}=\left\{\begin{array}{llllllll}
U_{1} & V_{1} & U_{2} & V_{2} & U_{3} & V_{3} & U_{4} & V_{4}
\end{array}\right\}^{\mathrm{T}}
\end{aligned}
$$

in which

$$
\Phi_{i}=\frac{1}{4}\left(1+\xi_{i} \xi\right)\left(1+\eta_{i} \eta\right)
$$

To treat the material inhomogeneity by using the GFEM, it may be written

$$
\Psi^{(e)}=\sum_{i=1}^{4} \Psi_{i} \Phi_{i}
$$

where $\Psi^{(e)}$ is the material property of the element.

Substituting Eq. (2.13) in Eq. (2.11) gives the strain matrix of element $(e)$ as

$$
\varepsilon^{(e)}=\mathbf{B} \Lambda^{(e)}
$$

where

$$
\mathbf{B}=\mathbf{L} \boldsymbol{\Phi}^{(e)}
$$

By imposing Eqs. (2.7), (2.13), (2.17) into Eq. (2.6), it can be achieved as

$$
\delta \boldsymbol{\Lambda}^{(e)^{\mathrm{T}}}\left(\iiint_{\Omega} \mathbf{B}^{\mathrm{T}} \mathbf{D B} d \Omega\right) \boldsymbol{\Lambda}^{(e)}+\delta \boldsymbol{\Lambda}^{(e)^{\mathrm{T}}}\left(\iiint_{\Omega} \rho \boldsymbol{\Phi}^{\mathrm{T}} \boldsymbol{\Phi} d \Omega\right) \ddot{\boldsymbol{\Lambda}}^{(e)}=0
$$


Since $\delta \boldsymbol{\Lambda}^{(e)^{\mathrm{T}}}$ is the variation of the nodal displacements and is arbitrary, it can be omitted from (2.19), thus this equation can be written as

$$
\mathbf{M}^{(e)} \ddot{\Lambda}^{(e)}+\mathbf{K}^{(e)} \boldsymbol{\Lambda}^{(e)}=\mathbf{0}
$$

where the mass and stiffness matrices are defined as

$$
\mathbf{M}^{(e)}=\iiint_{\Omega} \rho \boldsymbol{\Phi}^{\mathrm{T}} \mathbf{\Phi} d \Omega \quad \mathbf{K}^{(e)}=\iiint_{\Omega} \mathbf{B}^{\mathrm{T}} \mathbf{D B} d \Omega
$$

For finding the components of mass and stiffness matrices, the integral must be taken over the elements volume. As $\mathbf{D}$ and $\rho$ are not constant in the case of FG types distributions, these matrices are evaluated by numerical integration for each element using the Gauss-Legendre technique (Zienkiewicz, 2005). Now by assembling the element matrices, the global equations of motion for the FG-CNTRC disks can be obtained as

$$
\mathbf{M} \ddot{\Lambda}+\mathbf{K} \Lambda=\mathbf{0}
$$

Once the finite element equations are estimated, substituting $\boldsymbol{\Lambda}=\boldsymbol{\Lambda}_{0} \mathrm{e}^{\mathrm{i} \omega t}$ ( $\omega$ is the natural frequency) into Eq. (2.22) leads to an eigenvalue problem that can be solved using standard eigenvalue extraction procedures.

In this regard, for calculating the elemental characteristic matrices (2.21) with the use of Gauss-Legendre technique, obtaining the global ones and solve the eigenvalue problem after imposing the boundary conditions, a FE code is prepared by the authors.

\section{Numerical results and discussion}

\subsection{Validation}

To validate the current work, the data of a non-homogeneous solid circular plate with variable thickness can be used (Gupta et al., 2007). The outer radius of the disk is $r_{o}$ and the variable thickness is defined as

$$
h(r)=h_{0}\left(1+\alpha r+\beta r^{2}\right)
$$

where $h_{0}, \alpha$ and $\beta$ are the thickness at the middle and taper parameters, respectively. The elasticity modulus and density vary in the $r$ direction as below

$$
E(r)=E_{0} \mathrm{e}^{\mu r} \quad \rho(r)=\rho_{0} \mathrm{e}^{\eta r}
$$

in which $\mu$ and $\eta$ are non-homogeneity parameters.

The comparison of the first three frequency parameter $\left(\Omega=\omega \sqrt{\rho_{0} r_{0}^{2}\left(1-\nu^{2}\right) / E_{0}}\right)$ for a circular plate of $\eta=-0.5, \mu=0.1, \alpha=-0.5$ and $\beta=0.5$ with the published data is shown in Table 1 and a good agreement between these results is observed.

\subsection{Numerical results}

In this Section, the free vibrational response of FG-CNTRC disks with variable thickness is presented. The disk is made of Polymethyl-methacrylate (PMMA) as the matrix, where SWCNTs act as fibers aligned in the radial direction. The properties of basic materials are (Han and Elliott, 2007; Shen, 2009)

$$
\begin{array}{lll}
E^{m}=2.5 \mathrm{GPa} & \nu^{m}=0.34 & \rho^{m}=1150 \mathrm{~kg} / \mathrm{m}^{3} \\
E_{1}^{C N T}=5.6466 \mathrm{TPa} & E_{2}^{C N T}=7.08 \mathrm{TPa} & \nu^{C N T}=0.175 \\
\rho^{C N T}=1150 \mathrm{~kg} / \mathrm{m}^{3} & &
\end{array}
$$


Table 1. Frequency parameter compared with the result by Gupta (2007)

\begin{tabular}{|c|c|c|c|c|c|c|c|}
\hline \multirow{2}{*}{$\begin{array}{c}\text { Boundary } \\
\text { condition }\end{array}$} & \multicolumn{3}{|c|}{$h_{0}=0.1$} & \multicolumn{3}{c|}{$h_{0}=0.2$} \\
\cline { 2 - 8 } & GFEM & $\begin{array}{c}\text { Gupta } \\
(2007)\end{array}$ & $\begin{array}{c}\text { Difference } \\
{[\%]}\end{array}$ & GFEM & $\begin{array}{c}\text { Gupta } \\
(2007)\end{array}$ & $\begin{array}{c}\text { Difference } \\
{[\%]}\end{array}$ \\
\hline \hline \multirow{3}{*}{ Clamped } & $\Omega_{1}$ & 0.4121 & 0.4083 & 0.9 & 0.7765 & 0.7673 & 1.2 \\
\cline { 2 - 8 } & $\Omega_{2}$ & 1.4657 & 1.4471 & 1.2 & 2.5050 & 2.4604 & 1.8 \\
\cline { 2 - 8 } & $\Omega_{3}$ & 3.0403 & 2.9897 & 1.6 & 4.7195 & 4.6123 & 2.3 \\
\hline \multirow{2}{*}{$\begin{array}{l}\text { Simply } \\
\text { supported }\end{array}$} & $\Omega_{1}$ & 0.1857 & 0.1852 & 0.3 & 0.3650 & 0.3641 & 0.2 \\
\cline { 2 - 8 } & $\Omega_{2}$ & 1.0800 & 1.0715 & 0.8 & 1.9362 & 1.9258 & 0.5 \\
\cline { 2 - 8 } & $\Omega_{3}$ & 2.5635 & 2.5349 & 1.1 & 4.1489 & 4.1205 & 0.7 \\
\hline \multirow{3}{*}{ Free } & $\Omega_{1}$ & 0.3358 & 0.3348 & 0.3 & 0.6503 & 0.6480 & 0.3 \\
\cline { 2 - 8 } & $\Omega_{2}$ & 1.3553 & 1.3436 & 0.9 & 2.3823 & 2.3572 & 1 \\
\cline { 2 - 8 } & $\Omega_{3}$ & 2.9541 & 2.9159 & 1.3 & 4.7125 & 4.6299 & 1.8 \\
\hline
\end{tabular}

The key issue for successful application of the extended rule of mixture to CNTRCs is to determine the CNT efficiency parameter $\eta_{j}(j=1,2,3)$. However, there are no experiments conducted to determine the value of $\eta_{j}$ for CNTRCs (Shen, 2009). Han and Elliott (2007), with the use of MD simulation and energy minimization, obtained the elastic moduli of polymer/CNT composites. In the conventional rule of mixture, the whole system is assumed to be continuum and the interfaces between the matrix and fibers remain fully intact, thus the general macroscopic rule of mixtures cannot be applied straightforwardly to composites with strong interfacial interactions. Besides, micromechanics equations cannot capture the scale difference between the nano and micro levels. For this purpose, CNT efficiency parameters $\eta_{j}(j=1,2,3)$ are obtained by comparing Young's moduli $E_{1}^{C N T}$ and $E_{2}^{C N T}$ of CNTRCs achieved from the extended rule of mixture to those from MD simulation given by Han and Elliot (2007). It should be noticed that there are no MD results available for shear modulus $G_{12}$ in Han and Elliott (2007). The results are shown in Table 2 and will be used in the present study, in which it is assumed $\eta_{3}=0.7 \eta_{2}$ (Yas et al., 2013).

Table 2. Comparison of Young's moduli for polymer/CNTRC at $T_{0}=300$ (Yas et al., 2013)

\begin{tabular}{|c|c|c|c|c|c|c|}
\hline \multirow{2}{*}{$V_{C N T}^{*}$} & \multicolumn{2}{|c|}{ MD (Hanet al., 2007) } & \multicolumn{4}{|c|}{ Extended rule of mixture } \\
\cline { 2 - 7 } & $E_{1}[\mathrm{GPa}]$ & $E_{2}[\mathrm{GPa}]$ & $E_{1}[\mathrm{GPa}]$ & $\eta_{1}[-]$ & $E_{2}[\mathrm{GPa}]$ & $\eta_{2}[-]$ \\
\hline \hline 0.12 & 94.6 & 2.9 & 94.78 & 0.137 & 2.9 & 1.022 \\
\hline 0.17 & 138.9 & 4.9 & 138.68 & 0.142 & 4.9 & 1.626 \\
\hline 0.28 & 224.2 & 5.5 & 224.5 & 0.141 & 5.5 & 1.585 \\
\hline
\end{tabular}

Furthermore, the thickness profile of FG-CNTRC disk is in the form of

$$
h(r)=h_{0}\left(1-q\left(\frac{r}{b}\right)^{m}\right)
$$

where $h_{0}, q$ and $m$ are geometric parameters that $0 \leqslant q<1$ and $m>0$. By changing the values of $q$ and $m$, four different thickness profiles, namely constant, linear, concave and convex are introduced in Table 3 and, in the case of $a=0, b=0.5 m$, are shown in Fig. 4.

Table 3. Different kinds of thickness profiles

\begin{tabular}{|c|c|c|c|}
\hline Constant & Linear & Concave & Convex \\
\hline \hline \multirow{2}{*}{$q=0$} & $q=0.7$ & $q=0.7$ & $q=0.7$ \\
& $m=1$ & $m=0.5$ & $m=2$ \\
\hline
\end{tabular}




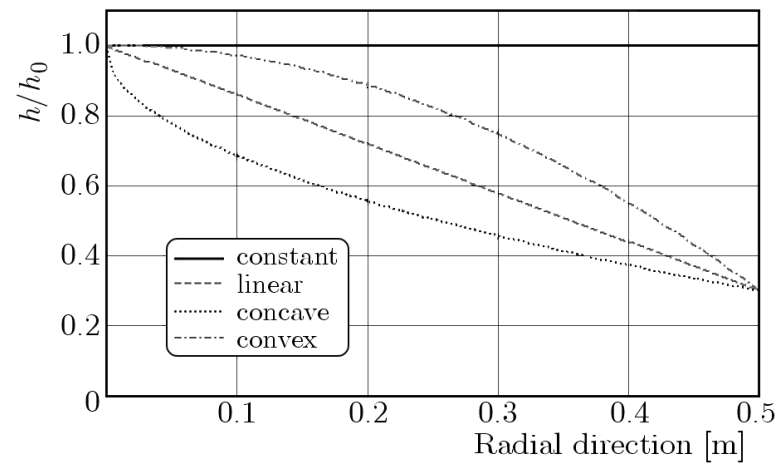

Fig. 4. Different kinds of thickness profiles

It should be also stated that the plane of revolution of the disk ( $r$-zplane) is devided into 1200 linear quadrilateral elements with mesh density of $60 \times 20$ regularly placed along the $r$ and $z$ direction. In the case of linear thickness profile, a schematic of finite element mesh is illustrated in Fig. 5.

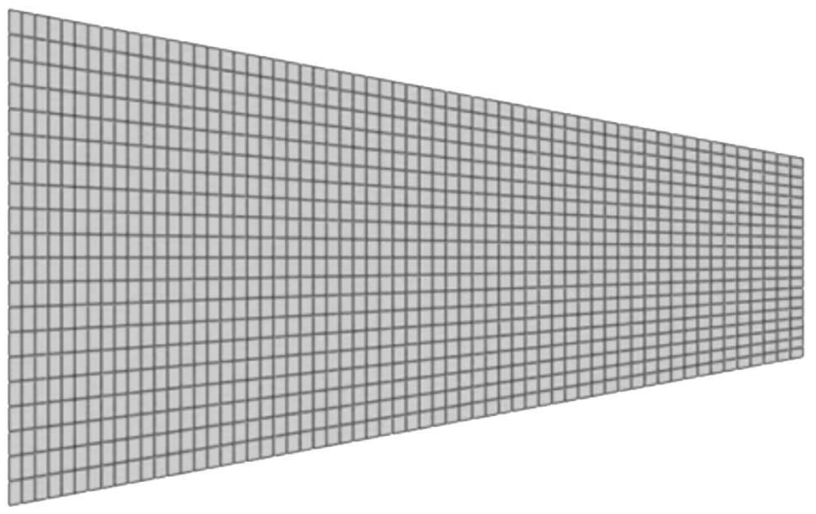

Fig. 5. Finite element mesh

Now, by introducing the normalized natural frequency as

$$
\Omega=\omega b \sqrt{\frac{\rho_{m}}{G_{m}}}
$$

the first five normalized natural frequencies for FG-CNTRC annular and solid disks for different types of distribution and volume fractions of CNTs, boundary conditions and thickness to radius ratios $\left(h_{0} / b\right)$ as well as different thickness profiles are presented.

The effect of volume fraction of CNTs $\left(V_{C N T}^{*}\right)$ on the first five normalized natural frequencies $(\Omega)$ of the solid UD and FG-CNTRC disk for different types of CNTs distribution is shown in Table 4 . In this case, the boundary conditions are assumed to be clamped, the thickness profile to be constant and $h_{0} / b=0.5$. As it can be seen; the normalized natural frequencies increase when $V_{C N T}^{*}$ raises due to growth of the structure stiffness. Moreover, changing the type of CNTs distribution, affects the magnitude of natural frequencies. The FG_V type of distribution has the highest natural frequencies and the FG_O has the lowest ones.

The comparison of first five normalized natural frequencies for UD and FG-CNTRC solid disks for different boundary conditions with the linear thickness profile, $V_{C N T}^{*}=0.12$ and $h_{0} / b=0.5$ is presented in Table 5 . According to the results, boundary conditions have noticeable effects on natural frequencies. Thus, by changing the boundary conditions, the free vibration response of the disk can be controlled. 
Table 4. Comparison of the first five normalized natural frequencies $(\Omega)$ for clamped UD and FG-CNTRC solid disks for different $V_{C N T}^{*}$ with constant thickness and $h_{0} / b=0.5$

\begin{tabular}{|c|c|c|c|c|c|c|c|c|c|c|c|c|}
\hline & \multicolumn{4}{|c|}{$V_{C N T}^{*}=0.12$} & \multicolumn{4}{|c|}{$V_{C N T}^{*}=0.17$} & \multicolumn{4}{|c|}{$V_{C N T}^{*}=0.28$} \\
\hline & $\overline{\mathrm{UD}}$ & FG_V & FG_O & FG_X & UD & FG_V & FG_O & FG_X & UD & FG_V & FG_O & FG_X \\
\hline$\overline{\Omega \Omega_{1}}$ & 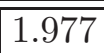 & $\overline{2.059}$ & 1.885 & 1.953 & 2.537 & $\overline{2.686}$ & 2.385 & 2.507 & 2.688 & 2.981 & 2.435 & 2.672 \\
\hline$\overline{\Omega_{2}}$ & 4.669 & 4.699 & 4.631 & 4.576 & 6.009 & 6.075 & 5.943 & 5.882 & 6.339 & 6.536 & 6.219 & 6.231 \\
\hline$\overline{\Omega_{3}}$ & 7.381 & 7.394 & 7.349 & 7.32 & 9.503 & 9.547 & 9.455 & 9.432 & 10.01 & 10.18 & 9.953 & 9.981 \\
\hline$\overline{\Omega_{4}}$ & 10.13 & 10.14 & 10.11 & 10.06 & 13.06 & 13.09 & 13.02 & 12.98 & 13.74 & 13.91 & 13.73 & 13.71 \\
\hline$\Omega_{5}$ & 12.94 & 12.94 & 12.67 & 12.42 & 16.54 & 16.54 & 16.03 & 15.64 & 17.02 & 16.70 & 16.49 & 16.25 \\
\hline
\end{tabular}

Table 5. Comparison of the first five normalized natural frequencies $(\Omega)$ for UD and FG-CNTRC solid disks for different boundary conditions with the linear thickness profile, $V_{C N T}^{*}=0.12$ and $h_{0} / b=0.5$

\begin{tabular}{|c|c|c|c|c|c|c|c|c|c|c|c|c|}
\hline & \multicolumn{4}{|c|}{ Clamped } & \multicolumn{4}{c|}{ Simply supported } & \multicolumn{4}{c|}{ Free } \\
\cline { 2 - 14 } & UD & FG_V & FG_O & FG_X & UD & FG_V & FG_O & FG_X & UD & FG_V & FG_O & FG_X \\
\hline \hline$\Omega_{1}$ & 1.44 & 1.53 & 1.323 & 1.426 & 1.317 & 1.427 & 1.125 & 1.335 & 2.942 & 3.009 & 2.775 & 2.723 \\
\hline$\Omega_{2}$ & 4.262 & 4.291 & 4.21 & 4.109 & 4.055 & 4.109 & 3.938 & 3.908 & 5.399 & 5.508 & 5.074 & 5.37 \\
\hline$\Omega_{3}$ & 6.936 & 6.957 & 6.884 & 6.818 & 6.669 & 6.709 & 6.581 & 6.579 & 7.945 & 8.085 & 7.658 & 7.905 \\
\hline$\Omega_{4}$ & 9.638 & 9.653 & 9.582 & 9.522 & 9.323 & 9.348 & 9.259 & 9.208 & 10.65 & 10.75 & 10.38 & 10.64 \\
\hline$\Omega_{5}$ & 12.4 & 12.41 & 12.35 & 12.33 & 12.03 & 12.04 & 11.98 & 11.96 & 13.41 & 13.49 & 10.79 & 13.39 \\
\hline
\end{tabular}

Table 6 demonstrates the effect of thickness-to-radius ratio on the normalized natural frequencies of UD and FG-CNTRC solid disks for different types of CNTs distribution with the concave thickness profile and $V_{C N T}^{*}=0.17$, and the boundary conditions assumed to be simply supported. It is obvious that enlarging the thickness-to-radius ratio leads to growth of natural frequencies, and this growth is larger in the lower natural frequencies.

Table 6. Comparison of the first five normalized natural frequencies $(\Omega)$ for simply supported UD and FG-CNTRC solid disks for different thickness-to-radius ratios $\left(h_{0} / b\right)$ with the concave thickness profile and $V_{C N T}^{*}=0.17$

\begin{tabular}{|c|c|c|c|c|c|c|c|c|c|c|c|c|}
\hline & \multicolumn{4}{|c|}{$h_{0} / b=0.1$} & \multicolumn{4}{c|}{$h_{0} / b=0.2$} & \multicolumn{4}{c|}{$h_{0} / b=0.3$} \\
\cline { 2 - 14 } & UD & FG_V & FG_O & FG_X & UD & FG_V & FG_O & FG_X & UD & FG_V & FG_O & FG_X \\
\hline \hline$\Omega_{1}$ & 0.473 & 0.562 & 0.337 & 0.475 & 0.881 & 1.032 & 0.638 & 0.889 & 1.205 & 1.382 & 0.905 & 1.215 \\
\hline$\Omega_{2}$ & 2.125 & 2.203 & 1.971 & 1.732 & 3.493 & 3.596 & 3.278 & 3.01 & 4.271 & 4.372 & 4.057 & 3.867 \\
\hline$\Omega_{3}$ & 4.175 & 4.154 & 4.065 & 3.866 & 6.284 & 6.313 & 6.124 & 6.053 & 7.349 & 7.407 & 7.184 & 7.182 \\
\hline$\Omega_{4}$ & 6.604 & 6.467 & 6.555 & 6.304 & 9.35 & 9.312 & 9.257 & 9.022 & 10.64 & 10.65 & 10.55 & 10.36 \\
\hline$\Omega_{5}$ & 9.355 & 9.113 & 9.366 & 9.059 & 12.65 & 12.56 & 12.63 & 12.41 & 14.11 & 14.07 & 14.08 & 13.95 \\
\hline
\end{tabular}

The influence of different thickness profiles on the normalized natural frequencies of free UD and FG-CNTRC solid disks with $V_{C N T}^{*}=0.28$ and $h_{0} / b=0.5$ for different types of CNTs distribution is illustrated in Table 7 . The achieved results show that the natural frequencies are highly dependent on the thickness profile. Moreover, the highest natural frequencies occur in the convex thickness profile and the smallest ones occur in the concave thickness profile.

Now, the free vibration response of FG-CNTRC annular disks of variable thickness is discussed. As before, the effect of volume fraction of CNTs, boundary condition, thickness to radius ratio and thickness profile on the first five normalized natural frequencies of annular UD and FG-CNTRC disks are studied, in this case of $a / b=0.2$. These results are illustrated in Tables 8-11. As it can be concluded from these tables, the normalized natural frequncies increase when 
Table 7. Comparison of the first five normalized natural frequencies $(\Omega)$ for free UD and FG-CNTRC solid disks for different thickness profiles with $h_{0} / b=0.5$ and $V_{C N T}^{*}=0.28$

\begin{tabular}{|c|c|c|c|c|c|c|c|c|c|c|c|c|}
\hline & \multicolumn{4}{|c|}{ Linear } & \multicolumn{4}{c|}{ Concave } & \multicolumn{4}{c|}{ Convex } \\
\cline { 2 - 14 } & UD & FG_V & FG_O & FG_X & UD & FG_V & FG_O & FG_X & UD & FG_V & FG_O & FG_X \\
\hline \hline$\Omega_{1}$ & 4.027 & 4.162 & 3.762 & 3.585 & 3.375 & 3.886 & 3.401 & 3.291 & 4.349 & 4.459 & 4.158 & 3.884 \\
\hline$\Omega_{2}$ & 7.38 & 7.566 & 6.942 & 7.257 & 7.014 & 7.211 & 6.479 & 6.896 & 7.793 & 7.95 & 7.482 & 7.65 \\
\hline$\Omega_{3}$ & 10.87 & 11.06 & 10.46 & 10.71 & 10.45 & 10.65 & 9.991 & 10.28 & 11.32 & 11.49 & 10.99 & 11.16 \\
\hline$\Omega_{4}$ & 14.49 & 14.67 & 14.17 & 14.42 & 14.04 & 14.23 & 13.71 & 13.97 & 14.95 & 15.13 & 14.64 & 14.89 \\
\hline$\Omega_{5}$ & 18.21 & 18.39 & 15.02 & 18.18 & 17.75 & 17.93 & 14.76 & 17.72 & 18.14 & 17.31 & 15.12 & 17.82 \\
\hline
\end{tabular}

Table 8. Comparison of the first five normalized natural frequencies $(\Omega)$ for clamped UD and FG-CNTRC annular disks for different $V_{C N T}^{*}$ with constant thickness and $h_{0} / b=0.5$

\begin{tabular}{|c|c|c|c|c|c|c|c|c|c|c|c|c|}
\hline & \multicolumn{4}{|c|}{$V_{C N T}^{*}=0.12$} & \multicolumn{4}{c|}{$V_{C N T}^{*}=0.17$} & \multicolumn{4}{c|}{$V_{C N T}^{*}=0.28$} \\
\cline { 2 - 14 } & UD & FG_V & FG_O & FG_X & UD & FG_V & FG_O & FG_X & UD & FG_V & FG_O & FG_X \\
\hline \hline$\Omega_{1}$ & 3.203 & 3.237 & 3.167 & 3.301 & 4.122 & 4.194 & 4.076 & 4.314 & 4.348 & 4.541 & 4.321 & 4.757 \\
\hline$\Omega_{2}$ & 6.598 & 6.609 & 6.589 & 6.513 & 8.493 & 8.535 & 8.485 & 8.419 & 8.951 & 9.108 & 8.977 & 9.015 \\
\hline$\Omega_{3}$ & 10.03 & 10.04 & 10.02 & 10.03 & 12.93 & 12.96 & 12.92 & 12.98 & 13.61 & 13.77 & 13.66 & 13.83 \\
\hline$\Omega_{4}$ & 13.09 & 13.13 & 12.86 & 12.55 & 16.66 & 16.67 & 16.34 & 15.82 & 17.21 & 17.06 & 16.81 & 16.47 \\
\hline$\Omega_{5}$ & 13.55 & 13.56 & 13.54 & 13.1 & 17.47 & 17.51 & 17.29 & 16.61 & 18.31 & 18.49 & 18.09 & 17.29 \\
\hline
\end{tabular}

Table 9. Comparison of first five normalized natural frequency $(\Omega)$ for UD and FG-CNTRC annular disks for different boundary conditions with linear thickness profile, $V_{C N T}^{*}=0.12$ and $h_{0} / b=0.5$

\begin{tabular}{|c|c|c|c|c|c|c|c|c|c|c|c|c|}
\hline & \multicolumn{4}{|c|}{ Clamped } & \multicolumn{4}{c|}{ Simply supported } & \multicolumn{4}{c|}{ Free } \\
\cline { 2 - 14 } & UD & FG_V & FG_O & FG_X & UD & FG_V & FG_O & FG_X & UD & FG_V & FG_O & FG_X \\
\hline \hline$\Omega_{1}$ & 2.829 & 2.838 & 2.801 & 2.928 & 2.228 & 2.264 & 2.124 & 2.337 & 3.205 & 3.234 & 3.066 & 2.945 \\
\hline$\Omega_{2}$ & 6.155 & 6.154 & 6.144 & 5.984 & 5.181 & 5.191 & 5.125 & 5.045 & 6.097 & 6.129 & 5.797 & 6.093 \\
\hline$\Omega_{3}$ & 9.478 & 9.477 & 9.456 & 9.422 & 8.178 & 8.14 & 8.152 & 8.129 & 9.232 & 9.215 & 8.944 & 9.196 \\
\hline$\Omega_{4}$ & 12.88 & 12.87 & 12.86 & 12.83 & 11.28 & 11.16 & 11.27 & 11.23 & 12.53 & 12.46 & 11.43 & 12.59 \\
\hline$\Omega_{5}$ & 16.37 & 16.36 & 16.35 & 15.49 & 14.47 & 14.26 & 13.39 & 14.49 & 15.93 & 15.09 & 12.28 & 15.16 \\
\hline
\end{tabular}

Table 10. Comparison of first five normalized natural frequency $(\Omega)$ for simply supported UD and FG-CNTRC annular disks for different thickness to radius ratio $\left(h_{0} / b\right)$ with concave thickness profile and $V_{C N T}^{*}=0.17$

\begin{tabular}{|c|c|c|c|c|c|c|c|c|c|c|c|c|}
\hline & \multicolumn{4}{|c|}{$h_{0}=0.1$} & \multicolumn{4}{c|}{$h_{0}=0.2$} & \multicolumn{4}{c|}{$h_{0}=0.3$} \\
\cline { 2 - 14 } & UD & FG_V & FG_O & FG_X & UD & FG_V & FG_O & FG_X & UD & FG_V & FG_O & FG_X \\
\hline \hline$\Omega_{1}$ & 1.429 & 1.193 & 1.418 & 1.432 & 2.212 & 2.023 & 2.156 & 2.329 & 2.593 & 2.515 & 2.488 & 2.781 \\
\hline$\Omega_{2}$ & 3.271 & 2.927 & 3.271 & 3.208 & 5.011 & 4.826 & 4.932 & 4.776 & 5.904 & 5.849 & 5.812 & 5.62 \\
\hline$\Omega_{3}$ & 5.712 & 5.279 & 5.767 & 5.592 & 8.421 & 8.209 & 8.418 & 8.285 & 9.626 & 9.561 & 9.609 & 9.559 \\
\hline$\Omega_{4}$ & 8.692 & 8.223 & 8.809 & 8.632 & 12.25 & 12.05 & 12.31 & 12.15 & 13.62 & 13.53 & 13.67 & 13.54 \\
\hline$\Omega_{5}$ & 12.14 & 11.69 & 12.31 & 12.12 & 16.37 & 16.19 & 16.48 & 16.34 & 17.81 & 17.64 & 17.76 & 17.83 \\
\hline
\end{tabular}


Table 11. Comparison of first five normalized natural frequency $(\Omega)$ for free UD and FG-CNTRC annular disks for different thickness profile with $h_{0} / b=0.5$ and $V_{C N T}^{*}=0.28$

\begin{tabular}{|c|c|c|c|c|c|c|c|c|c|c|c|c|}
\hline & \multicolumn{4}{|c|}{ Linear } & \multicolumn{4}{c|}{ Concave } & \multicolumn{4}{c|}{ Convex } \\
\cline { 2 - 14 } & UD & FG_V & FG_O & FG_X & UD & FG_V & FG_O & FG_X & UD & FG_V & FG_O & FG_X \\
\hline \hline$\Omega_{1}$ & 4.392 & 4.448 & 4.187 & 3.881 & 4.077 & 4.149 & 3.798 & 3.56 & 4.752 & 4.775 & 4.628 & 4.229 \\
\hline$\Omega_{2}$ & 8.336 & 8.399 & 7.978 & 8.281 & 7.928 & 7.986 & 7.472 & 7.887 & 8.814 & 8.858 & 8.585 & 8.731 \\
\hline$\Omega_{3}$ & 12.58 & 12.59 & 12.27 & 12.48 & 12.11 & 12.09 & 11.75 & 12.01 & 13.11 & 13.13 & 12.86 & 13.01 \\
\hline$\Omega_{4}$ & 17.03 & 16.98 & 15.97 & 17.09 & 16.54 & 16.44 & 15.69 & 16.61 & 17.56 & 16.91 & 16.07 & 17.63 \\
\hline$\Omega_{5}$ & 21.58 & 19.22 & 16.79 & 21.35 & 21.13 & 20.97 & 16.31 & 21.22 & 18.63 & 17.57 & 17.31 & 18.79 \\
\hline
\end{tabular}

the volume fraction of CNTs or thickness-to-radius ratio raises. Moreover, the boundary condition and the type of CNTs distribution as well as the thickness profile have a great influence on the normalized natural frequencies. Besides, in comparison to solid disks, annular disks of the same conditions have larger normalized natural frequencies and, unlike the solid disks, the highest normalized natural frequencies of annular disks occur in the case of FG_X type of CNTs distribution.

Thus, the normalized natural frequencies can be controlled and altered by changing the distribution and volume fraction of CNTs, boundary condition, thickness-to-radius ratio and the thickness profile.

\section{Conclusions}

In this study, free vibration analysis of thick FG-CNTRC annular and solid disks with variable thickness is presented. The axisymmetric conditions are assumed, volume fraction of CNTs is considered to be graded continuously along the radial direction and material properties are estimated through the extended rule of mixture. By employing the graded finite element method and Hamilton's principle, the free vibrational response of FG-CNTRC disks is investigated. Detailed parametric studies are performed to illustrate the effects of several parameters including the type of distribution and volume fraction of CNTs, boundary condition, thickness-to-radius ratio and the thickness profile on the normalized natural frequencies of FG-CNTRC disks. From this analysis, some typical conclusions can be made:

- The normalized natural frequencies increase when the CNTs volume fraction or thickness-to-radius ratio rises.

- In solid FG-CNTRC disks, the FG_V type of CNTS distribution has higher normalized natural frequencies than the other types, and in the case of annular FG-CNTRC disks, the higher normalized frequencies occur in FG_X type of CNTs distribution.

- By using the variable thickness profile, the normalized natural frequencies can be controlled. Convexing the thickness profile generates larger normalized natural frequencies and concaving the thickness profile, generates smaller ones.

\section{References}

1. Alipour M.M., Shariyat M., Shaban M., 2010, A semi-analytical solution for free vibration of variable thickness two-directional-functionally graded plates on elastic foundations, International Journal of Mechanics and Material in Design, 6, 293-304

2. Ashrafi H., Asemi K., Shariyat M., Salehi M., 2013, Two-dimensional modelling of heterogeneous structures using graded finite element and boundary element methods, Meccanica, 48, $663-680$ 
3. DAi H., 2006, Carbon nanotubes: opportunities and challenges, Surface Science, 500, 218-241

4. Efraim E., Eisenberger M., 2007, Exact vibration analysis of variable thickness thick annular isotropic and FGM plates, Journal of Sound and Vibration, 299, 720-738

5. Esawi A.M.K., FArag M.M., 2007, Carbon nanotube reinforced composites: potential and current challenges, Materials and Design, 28, 2394-2401

6. Fiedler B., Gojny F.H., Wichmann M.H.G., Nolte M.C.M., Schulte K., 2006, Fundamental aspects of nano-reinforced composites, Composites Science and Technology, 66, 3115-3125

7. Gupta U.S., Lal R., Sharma S., 2007, Vibration of non-homogeneous circular Mindlin plates with variable thickness, Journal of Sound and Vibration, 302, 1-17

8. Han Y., Elliott J., 2007, Molecular dynamics simulations of the elastic properties of polymer/carbon nanotube composites, Computational Materials Science, 39, 315-323

9. Hu N., Fukunaga H., Lu C., Kameyama M., Yan B., 2005, Prediction of elastic properties of carbon nanotube reinforced composites, Proceeding of the Royal Society A, 461, 1685-1710

10. Kang I., Heung Y., Kim J., Lee J., Gollapudi R., et Al., 2006, Introduction to carbon nanotube and nanofiber smart materials, Composites Part B, 37, 382-394

11. Ke L.L., Yang J., Kitipornchai S., 2010, Nonlinear free vibration of functionally graded carbon nanotube-reinforced composite beams, Composite Structures, 92, 676-683

12. Kim J.H., Paulino G.H., 2002, Isoparametric graded finite elements for nonhomogeneous isotropic and orthotropic materials, Journal of Applied Mechanics, 69, 502-514

13. Koizumi M., 1993, The concept of FGM, Ceramic Transactions, Functionally Gradient Materials, 34, 3-10

14. Lau K.T., Gu C., Hui D., 2006, A critical review on nanotube and nanotube/nanoclay related polymer composite materials, Composites Part B, 37, 425-436

15. Odegard G.M., Gates T.S., Nicholson L.M., Wised K.E., 2002, Equivalent-continuum modeling of nano-structured materials, Composites Science and Technology, 62, 1869-1880

16. Odegard G.M., Gates T.S., Wise K.E., Park C., Siochi E.J., 2003, Constitutive modeling of nanotube-reinforced polymer composites, Composites Science and Technology, 63, 1671-1687

17. Shen H.S., 2009, Nonlinear bending of functionally graded carbon nanotube reinforced composite plates in thermal environments, Composite Structures, 91, 9-19

18. Sobhani Aragh B., Yas M.H., 2010, Static and free vibration analyses of continuously graded fiber-reinforced cylindrical shells using generalized power-law distribution, Acta Mechanica, 215, $155-173$

19. Tajeddini V., Ohadi A., 2011, Three-dimensional vibration analysis of functionally graded thick, annular plates with variable thickness via polynomial-Ritz method, Journal of Vibration and Control, 18, 1698-1707

20. Thostenson E.T., Ren Z.F., Chou T.W., 2001, Advances in the science and technology of carbon nanotubes and their composites: a review, Composites Science and Technology, 61, 1899-1912

21. Yas M.H., Pourasghar A., Kamarian S., Heshmati M., 2013, Three-dimensional free vibration analysis of functionally graded nanocomposite cylindrical panels reinforced by carbon nanotube, Materials and Design, 49, 583-590

22. Zafarmand H., Hassani B., 2014, Analysis of two-dimensional functionally graded rotating disks with variable thickness, Acta Mechanica, 225, 453-464

23. Zafarmand H., Kadkhodayan M., 2015, Nonlinear analysis of functionally graded nanocomposite rotating thick disks with variable thickness reinforced with carbon nanotubes, Aerospace Science and Technology, 41, 47-54 
24. Zhu R., Pan E., Roy A.K., 2007, Molecular dynamics study of the stress-strain behavior of carbon-nanotube reinforced Epon 862 composites, Material Science and Engineering: A, 447, 51-57

25. Zhu P., Lei Z.X., Liew K.M., 2012, Static and free vibration analyses of carbon nanotube reinforced composite plates using finite element method with first order shear deformation plate theory, Composite Structures, 94, 1450-1460

26. Zienkiewicz O.C., TAylor R.L., 2005, The Finite Element Method for Solid and Structural Mechanics, Elsevier Butterworth-Heinemann, Oxford

Manuscript received November 1, 2013; accepted for print June 1, 2015 